\title{
Single Framework Recombinant Antibody Fragments Designed for Protein Chip Applications
}

\author{
Cornelia Steinhauer, Christer Wingren, Ann-Christin Malmborg Hager, and Carl A.K. Borrebaeck \\ Department of Immunotechnology, Lund University, Lund, Sweden
}

BioTechniques 33:S38-S45 (December 2002)

\section{ABSTRACT}

High-throughput proteomics, based on the microarray platform, requires stable, highly functional components that will yield a highly sensitive read-out of low abundance proteins. Although antibodies are the best characterized binding molecules for this purpose, only a fraction of them appear to behave satisfactorily in the chip format. Therefore, high demands need to be placed on their molecular design. In the present study, we have focused on recombinant antibody design based on a single framework for protein chip applications, aiming at defining crucial molecular probe parameters. Our results show that engineered human recombinant scFv antibody fragments that displayed appropriate biophysical properties (molecular [functional] stability in particular) can be generated, making them prime candidates for high-density antibody arrays. In fact, a superior framework that displays both multifaceted adsorption properties and very high functional stability over several months on chips (stored in a dried-out state) was identified. Taken together, designed scFv fragments based on a single molecular scaffold, readily accessible in large phage display libraries, can undoubtedly meet the requirements of probe content in antibody microarrays, particularly for global proteome analysis.

\section{INTRODUCTION}

The term "microarray analysis" implies a highly multiplexed procedure, in which all relevant parameters to a particular biological process are screened simultaneously. In genomics, highdensity DNA microarrays are well established, allowing simultaneous analysis of entire genomes (23). The power of these transcriptional profiling screens has been demonstrated in a number of applications, such as stratification of various cancers $(1,8,10)$ and characterization of other biological events $(9,19,30)$. Establishing a protein microarray procedure that could globally analyze the protein content of various tissues or cells could close the existing information gap between genomics and proteomics $(2,13,24)$. For comparative proteome screening of healthy and disease tissue, such microarrays will require the use of a "probe" on the surface of a chip that can "catch" native and posttranslationally modified proteins. Anti- bodies are obvious candidates for catcher probes, because of their potentially exquisite specificity and well-characterized binding paratopes.

For dedicated arrays (i.e., arrays containing 15 to 20 different antibody specificities covering, for example, a signaling pathway, specific pro-inflammatory cytokines, or kinases), antibodies can be selected and directly tested for on-chip functionality. However, since only a fraction of all antibodies can be used for chip applications (14), this approach can only be used for small arrays. Thus, the next step to take in antibody microarrays is a more global analysis of the proteome, where 1000 to 5000 proteins can be analyzed using this multiplexing principle. The catcher probes used for such an approach would have to be based on recombinant antibodies and phage display libraries, containing several billion antibody members $(25,31,33)$. Because the antibodies cannot be synthesized on the surface of the chips, as is done for DNA arrays, the probes have to be spotted onto the chip surface in an array format and coupled to a downstream, high-throughput detection system. In such global analysis, a test of each antibody fragment for its on-chip performance is virtually impossible and the probes have to be directly designed for the array application, thus ensuring a highly functional analysis.

In the present investigation, we have studied a particular antibody design based on a single framework for protein chip applications aimed at defining crucial molecular parameters for microarrays. Antibody libraries built on a single framework have the advantage that one molecular scaffold can be selected that directly fulfills particular requirements of a certain application, such as stability, immunogenicity, bacterial productivity, and so forth (31). Our results demonstrate that single framework antibody fragments (sinFabs) display on-chip stability and sensitivity that make them very useful as probes for protein chip applications of all kinds. Consequently, in the not-too-distant future, we will likely be able to analyze the proteome using these types of probes, in a similar manner to what is presently possible for the genome. There is, however, no doubt that this task is more difficult because proteomes are less well defined, and bioinformatics will therefore continue to be a significant challenge. 


\section{MATERIALS AND METHODS}

\section{Antibodies}

The scFv clone CT17, selected from the recombinant human scFv antibody library, n-CoDeR (31), and kindly provided by BioInvent Therapeutics (Lund, Sweden), was produced in Escherichia coli, and soluble scFv molecules were retrieved from the periplasmic fraction and further purified by affinity chromatography on $\mathrm{Ni}^{2+}$-NTA (Qiagen, Hilden, Germany). The scFv clones ITC33-ICZ $/ 33$ [denoted ITC33; derived from the intact human monoclonal antibody ITC33 (28)], ITC52 [denoted ITC52; derived from the intact human monoclonal antibody ITC52 (28)], and ITC88-F3c10/2 [denoted ITC88 (22)], kindly provided by Drs. M. Ohlin and J. Lantto (Dept. of Immunotechnology, Lund University, Lund, Sweden), were produced in Pichia pastoris. Soluble scFv molecules were purified from crude expression supernatants by ion-exchange chromatography on SP Sepharose ${ }^{\mathrm{TM}}$ Fast Flow (Amersham Biosciences, Uppsala, Sweden) followed by HPLC-SE chromatography on Superdex 75 prep grade (Amersham). The activity/specificity and purity of the scFv preparations were confirmed by the means of antigen-specific ELISA, antigen-specific dot-blot analysis (Figure 2), and 12\% SDS-PAGE and Western blot analysis, respectively.

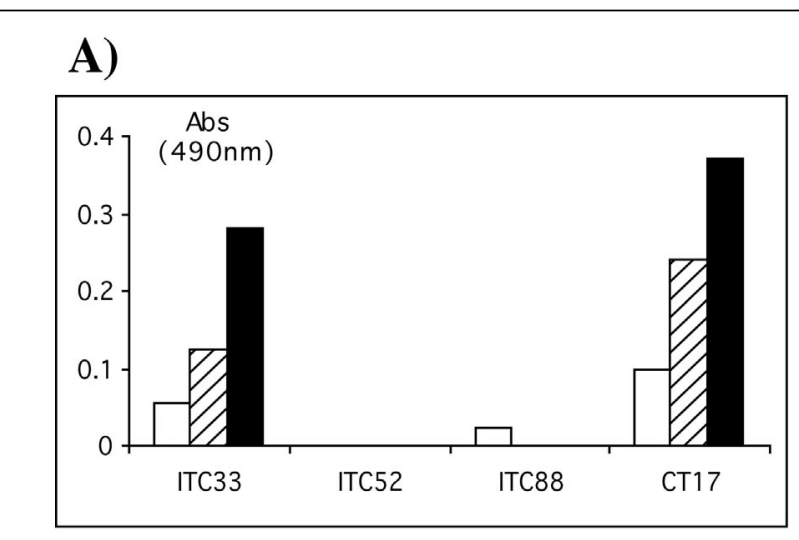

B)

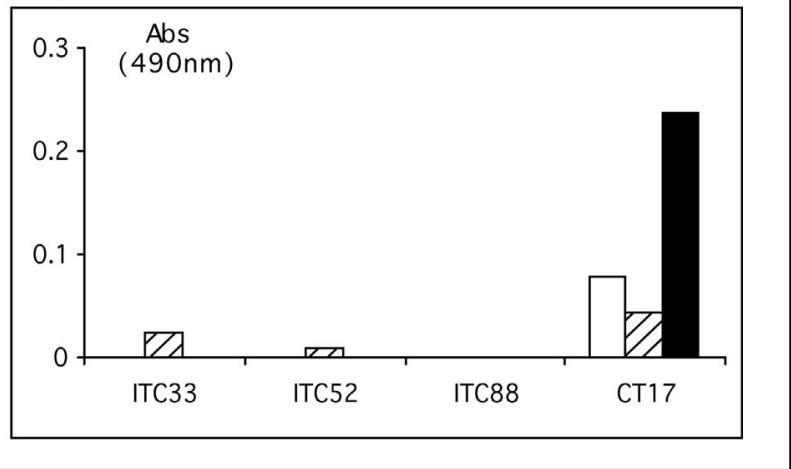

Figure 1. Comparison of the adsorption properties of $s c F v$ molecules based on different frameworks and spotted onto three different solid supports: polystyrene plastic (open bars), silicon (striped bars), and nitrocellulose-coated silicon (black bars). (A) Detection of adsorbed scFv molecules. (B) Detection of adsorbed and functionally active $\mathrm{scFv}$ molecules.

\section{Adsorption Studies}

Polystyrene silicon chips (Costar 3590 High-Capacity Polystyrene 96-well plate; Corning Costar, Corning NY, USA) and nitrocellulose-coated silicon chips (3) were used as solid supports. The supports were incubated with $10 \mathrm{ng} \mathrm{scFv}$ (clones CT17, ITC33, ITC52, or ITC88) $/ \mathrm{mm}^{2}$ surface in $0.1 \mathrm{M}$ sodium carbonate, $\mathrm{pH} 9.6$, overnight at $4^{\circ} \mathrm{C}$. Residual binding sites were blocked with $5 \%$ milk powder. Adsorbed scFv fragments were detected by using the anti-FLAG M2 mouse monoclonal antibody at $1 \mu \mathrm{g} / \mathrm{mL}$ (Sigma, St. Louis, MO, USA) and horseradish peroxidase-conjugated rabbit anti-mouse Ig diluted 1/2000 (Dako A/S, Glostrup, Denmark). Ortho-phenyldiamine (OPD) was used as enzyme chromogene and the absorbance was determined at $490 \mathrm{~nm}$ (Figure 1A). Adsorbed, functionally active $\mathrm{scFv}$ was detected by adding the appropriate antigen, i.e., biotinylated cholera toxin subunit B (for clone CT17; Sigma) or recombinant soluble $\mathrm{gB}$ produced in Chinese hamster ovary cells (ITC33, ITC52, and ITC88), kindly provided by Dr. C. Méric (Aventis Pasteur, Marcy-l'Etoile, France) (29). Biotinylated antigen was detected by using horseradish peroxidase-conjugated avidin diluted 1/10000 (Dako). gB was detected by using anti-gB mouse monoclonal antibody 5815 , kindly provided by Dr. M. Mach (The Fridrich-Alexander Universitat, Ehrlangen, Germany), diluted 1/500 and horseradish peroxidase-conjugated rabbit anti-mouse Ig diluted 1/2000 (Figure 1B). In both cases, OPD was used as enzyme chromogene and the absorbance was determined at $490 \mathrm{~nm}$.

\section{Functional On-Chip Stability}

Duplicates of $1 \mu \mathrm{L}$ of scFv fragments $(1 \mu \mathrm{g} / \mu \mathrm{L}$ of clones CT17, ITC33, ITC52, or ITC88) in phosphate-buffered saline (PBS), pH 7.4, were manually spotted onto separate nitrocellulose membranes $(0.45 \mu \mathrm{m}$; Bio-Rad, Solna, Sweden) in numerous replicates on day 0 , allowed to dry out, and subsequently stored in a dessicator at $4^{\circ} \mathrm{C}$. Every third or fourth day, the functional stability of the stored $\mathrm{scFv}$ membranes was evaluated by adding the appropriate antigen, biotinylated cholera toxin subunit B (CT17) or recombinant soluble gB (ITC33, ITC52, and ITC88). Biotinylated antigen was detected by using horseradish peroxidase-conjugated avidin diluted 1/10000. gB antigen was detected by using anti-gB mouse monoclonal antibody 5815 , diluted 1/500, and horseradish peroxidase-conjugated rabbit anti-mouse Ig diluted 1/2000. In both cases, the color reaction was developed with an ECL+ substrate kit (Amersham Biosciences AB, Uppsala, Sweden) and spot density was quantified in a GS-710 densitometer (Bio-Rad). The predicted half-life $\left(\mathrm{t}^{\mathrm{P}}{ }_{1 / 2}\right.$, dotted line) was estimated based on the trend lines (solid lines) to be for CT17: > 6 months; ITC33: 7 days; ITC52: 42 days; and ITC88: 39 days (Figure 2).

\section{Structural Analysis}

Structural homology models were generated using Web Antibody Modelling (WAM) (http://antibody.batn.ac.uk). Residues involved in the VH-VL interface interactions were identified using Endscript (http://prodes.toulouse.inra.fr/ESPript/ENDscript/ endscript.html), SwissPDBviewer (11), and WebLab ${ }^{\mathrm{TM}}$ Viewer 
(Accelrys, San Diego, CA, USA). Figure 3 was prepared using WebLab Viewer. Buried surface area (area of interaction between VH and VL) was computed with MS (7) and Surfv (27).

\section{DSC Analysis}

DSC analysis was performed on a VP-DSC (Microcal, Northampton, MA, USA). The measurements were performed in PBS, $\mathrm{pH} 7.4$, at a protein concentration of 0.1 or $0.2 \mathrm{mg} / \mathrm{mL}$, with PBS in the reference cell.

\section{RESULTS AND DISCUSSION}

We have focused on the recombinant antibody design based on a single framework (31) in order to gain further insight into important biophysical parameters, in particular molecular stability, for probes intended for protein chip applications. To this end, human recombinant $s c F v$ antibody fragments based on four common frameworks, such as VH3-23/VL1-47 (clone CT17), VH5-51/VL2-23 (clone ITC33), VH5-51/VKIIIb (clone ITC52), and VH3-30/VKIIIa (clone ITC88) were selected as probes (Table 1). The frameworks, two carrying lambda light chains and two carrying kappa light chains, were selected in part for their ability to yield high expression of scFvs, a molecular parameter that is correlated with molecular stability (35). In addition, we have recently shown that $\mathrm{scFv}$ probes, based on VH3-23/VL1-47 selected from the n-CoDeR library (31), can provide specific and sensitive detection in the 600 -amol range in a microarray format using mass spectrometry analysis (3). The individual $s c F v$ clones used in the present study were all selected against a protein antigen, either cholera toxin subunit B (CT17) or various epitopes on glycoprotein B of human cytomegalovirus (ITC33, ITC52, and ITC88). Consequently, the overall topography of their antigen-binding sites would be rather similar (32), thereby minimizing the non-framework related differences between the model probes.

The primary structure of the four model frameworks was compared initially (Table 1). The frameworks, and the individual domains thereof, display a high sequence homology $(>55 \%)$. The $\mathrm{pI}$ values vary from 4.7 (ITC33) to 6.8 (CT17), but are lower than the $\mathrm{pH}$ value of the working buffer (PBS, $\mathrm{pH} 7.4$ ) in all four cases. Hence, all frameworks will be negatively charged when applied to the chips, with ITC33 having the most negative change. Furthermore, the frameworks also display differences in hydrophobicity (expressed as hydropathicity values), with CT17 having the most hydrophobic framework. Interestingly, the aliphatic index (15) indicates that the thermostability of the frameworks differs, i.e., CT17 and ITC88 would have the most stable frameworks while ITC33 has the least stable one. Moreover, the instability index (12) im- plies that only the framework of CT17 would be defined as a stable scaffold. Based on the amino acid sequence of framework residues H6, H7, and $\mathrm{H} 10$ (18), scFv clones CT17 and ITC88 can be classified as type II molecules. It is worth noting that experimental observations have shown that most "well-behaved" antibodies (high production yields, good refolding yields in vitro, high thermostability) actually belong to type II (18). Taken together, the primary structure comparison indicates that the frameworks of CT17 (VH3-23/VL1-47) and ITC88 (VH3-30/VKIIIa) have the highest predicted molecular stability, making them prime candidates as probes for protein chip applications.

To experimentally identify crucial biophysical probe parameters, the adsorption properties of the frameworks were then compared (Figure 1). Potential probes should ideally display multifaceted adsorption properties to allow a variety of surfaces to be utilized, since this approach to depositing probes is highly dependent on the target surface properties $(3,5)$. Three common solid supports (polystyrene plastic, silicon, and nitrocellulosecoated silicon) were used and parallel experiments were run to distinguish between only adsorbed $s c F v$ (Figure $1 \mathrm{~A}$ ) and $\mathrm{scFv}$ adsorbed in a functionally active state (Figure 1B). While ITC33 was readily adsorbed to all three supports (Figure 1A), molecules in an active state could only be detected on silicon chips (Figure 1B). The results indicate that the adsorption of ITC33 eliminated most, if not all, antigen-binding activity, either by sterical hindrance (due to the orientation of the adsorbed molecules) or by a partial or complete collapse of the framework. Recent experiments have shown that only $20 \%$ of arrayed intact antibodies retained their activity (14), indicating that the loss of biological activities of spotted probes may be an inherent problem. In the case of ITC52 and ITC88, either no signals or only weak ones were detected for both adsorption assays (Figure 1A, B). These results suggest that these frameworks were poorly adsorbed to these particular supports or subjected to a deleterious phenomenon similar to that observed for ITC33.

In contrast to the above frameworks, CT17 showed multifaceted adsorption properties. It not only readily adsorbed to all three

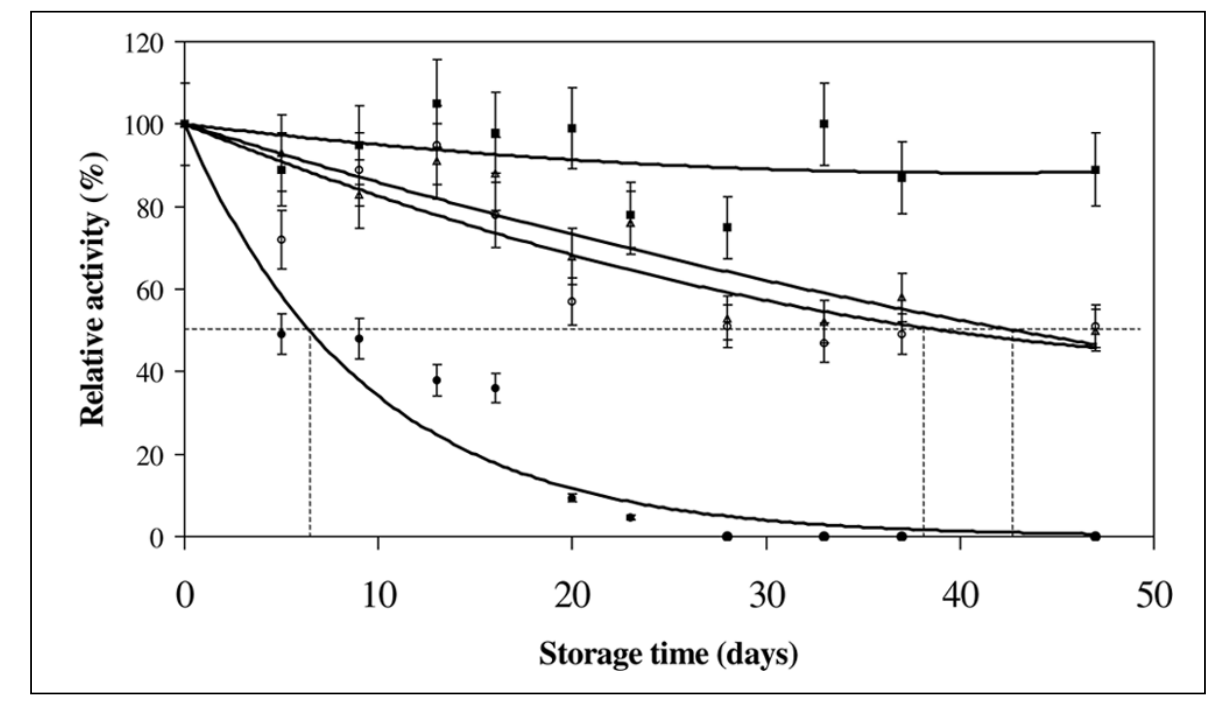

Figure 2. Comparison of the functional on-chip stability of $s c F v$ molecules based on different frameworks, spotted onto a solid support (nitrocellulose), and stored dry at $4^{\circ} \mathrm{C}$. CT17: solid squares; ITC33: solid circles; ITC52: open triangles; and ITC88: open circles. 


\section{RESEARCH REPORT}

supports (Figure 1A) but also displayed a retained antigen-binding activity (Figure 1B). In accordance with recent data (3), best adsorption/activity (highest signals) were obtained using a nitrocellulose-coated silicon surface. While the results indicate that lambda frameworks (ITC33, CT17) have superior adsorption properties compared to kappa frameworks (ITC52, ITC88) (Figure 1A), there is no direct correlation between the adsorption process and any single biophysical parameter, such as charge or hydrophobicity (Table 1, Figure 1A). Interestingly, the retained (clone CT17) or lost (clone ITC33) binding activity of adsorbed scFv molecules correlated well with the predicted stability of these frameworks based on primary structure analysis (Table 1, Figure 1B).

To confirm that the superior adsorption behavior displayed by $\mathrm{CT} 17 \mathrm{scFv}$ was not a clone-specific phenomenon, two additional scFv clones based on the VH3-23/VL1-47 framework were selected from the n-CoDeR library (31) and analyzed. To further generalize the observations, we also selected two nonprotein-specific scFv clones; FITC8 (directed against the hapten fluoresceine) and sMUC159 (directed against a mucine-1 derived peptide). These two scFvs carried other antigen-binding site geometries (cavity, groove) in addition to the planar $(31,32)$. The adsorption properties of both FITC8 and sMUC159 scFvs were similar to those of CT17 (data not shown). These results further strengthen the observation that scFvs based on the VH323/VL1-47 framework are strong protein array probe candidates.

The issue of functional probe stability of protein chips has been cited frequently as one of the major obstacles to successful protein chip development $(13,14,26)$. Probes are usually arrayed in picoliter scale, meaning that the spotted drops would dry out within 1-2 seconds, which often leads to the denaturation and inactivation of proteins. Furthermore, long-term storage of protein chips-a vital feature from a manufacturing and practical point of view-has been questioned for similar reasons. Consequently, high demands are placed on the molecular (functional) stability of the probes to achieve any of the desired goals for high-density antibody microarrays.

To address the issue of molecular probe stability, we decided to evaluate the on-chip stability of the four frameworks (Figure 2 ), since this stability is directly correlated to long-term storage and also to general performance of the chips during proteome analysis. On day 0 , the scFvs were spotted onto ordinary nitrocellulose membranes, a solid support to which all frameworks were found to be adequately adsorbed in a functional orientation (data not shown). The probes were allowed to dry out completely and the chips were then stored at $4^{\circ} \mathrm{C}$ in a dessicator. The functional stability (the retained binding capacity) of the dried scFvs was determined every third or fourth day by adding the appropriate antigen. Results showed that the on-chip stability of the model frameworks differed significantly (Figure 2). While the activity of scFv ITC33 dropped immediately, ITC52, ITC88, and CT17 were active to different degrees even after 47 days. Of note, CT17 retained about $90 \%$ of its original antigenbinding activity on day 47 . The predicted half life $\left(\mathrm{t}^{\mathrm{P}}{ }_{1 / 2}\right)$ was estimated to be more than 6 months for CT17, 42 days for ITC52, 39 days for ITC88, and only 7 days for ITC33. Interestingly, similar results were obtained whether the chips were stored at $4^{\circ} \mathrm{C}$ or at room temperature (data not shown). The superior probe stability displayed by CT17 was further corroborated by analysis of two additional $\mathrm{scFv}$ clones based on the

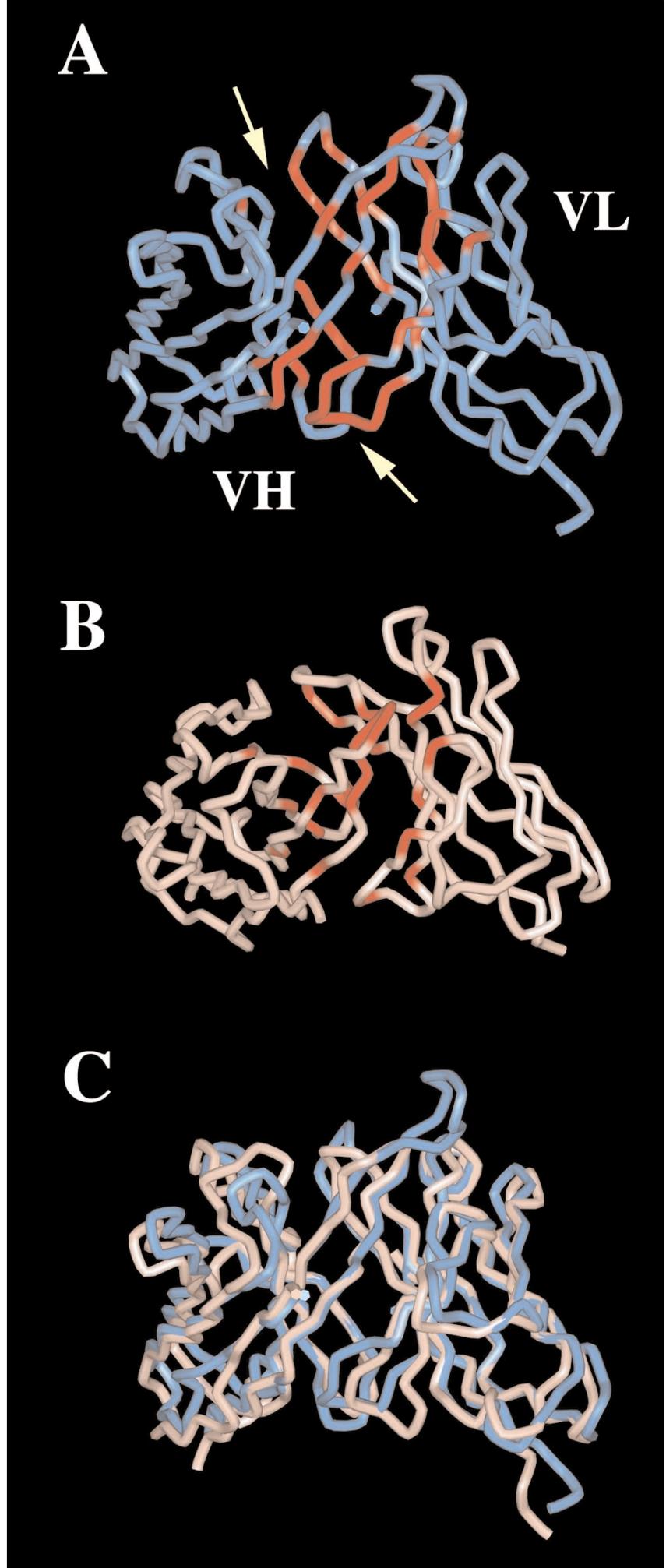

Figure 3. Comparison of the $\mathrm{VH}-\mathrm{VL}$ interface of scFv molecules, displaying the highest (CT17) and lowest (ITC33) on-chip stability. (A) Side view of the VH-VL domains of CT17. Residues involved in the VH-VL interface interactions are red. (B) Side view of the VH-VL domains of ITC33. Residues involved in VH-VL interface interactions are red. (C) Side view of the VH-VL domains of CT17 (blue) and ITC33 (pink). The structures were overlapped by superimposition of the structurally conserved core of the $\mathrm{Fv}$ unit (6). 
Table 1. Comparison of the Primary Structure of scFv Antibodies Based on Different Frameworks

\begin{tabular}{|c|c|c|c|c|c|c|c|c|c|c|}
\hline \multirow{3}{*}{$\begin{array}{l}\text { Framework } \\
\text { (VH-VL) }\end{array}$} & \multirow[b]{3}{*}{ Clone } & \multicolumn{9}{|c|}{ Primary Structure Comparison } \\
\hline & & \multirow{2}{*}{$\begin{array}{l}\text { No. of } \\
\text { residues }\end{array}$} & \multicolumn{3}{|c|}{ Framework homologya } & \multirow[b]{2}{*}{ plb } & \multirow{2}{*}{$\begin{array}{l}\text { Hydro- } \\
\text { pathicityc }\end{array}$} & \multirow{2}{*}{$\begin{array}{l}\text { Aliphatic } \\
\text { indexd }\end{array}$} & \multirow{2}{*}{$\begin{array}{l}\text { Instability } \\
\text { index }\end{array}$} & \multirow{2}{*}{$\begin{array}{c}\text { Classification } \\
\text { of } \mathrm{VH}^{\mathbf{f}}\end{array}$} \\
\hline & & & VH-VL (\%) & VH (\%) & VL (\%) & & & & & \\
\hline VH3-23 / VL1-47 & CT17 & 274 & 100 & 100 & 100 & 6.8 & -0.29 & 68 & 36 & II \\
\hline VH5-51 / VL2-23 & ITC33 & 257 & 68 & 55 & 81 & 4.7 & -0.43 & 56 & 42 & III \\
\hline VH5-51 / VKIIIb & ITC52 & 259 & 60 & 62 & 59 & 5.6 & -0.53 & 60 & 45 & III \\
\hline VH3-30 / VKIIIa & ITC88 & 255 & 72 & 87 & 57 & 6.2 & -0.33 & 70 & 47 & II \\
\hline \multirow{2}{*}{\multicolumn{11}{|c|}{$\begin{array}{l}\text { aSequence homology between the different frameworks, not including the CDR regions, link } \\
\text { C-terminal FLAG-tag and a His }{ }_{6} \text {-tag, while ITC33, ITC52 and ITC88 only carries a C-termina } \\
\text { bThe theoretical pl value was calculated using the ProtParam tool (http://www.expasy.org). }\end{array}$}} \\
\hline & & & & & & & \\
\hline \multicolumn{11}{|c|}{ cGrand average of hydrophathicity (20). A lower value (more negative) implies an overall lower hydrophobicity. } \\
\hline \multirow{2}{*}{\multicolumn{11}{|c|}{$\begin{array}{l}\text { dA statistical index, defined as the relative volume of a protein occupied by aliphatic side chains and should be seen as a positive fac- } \\
\text { tor for the increase of thermostability of globular proteins (15). } \\
\text { eA statistical index, based on the observation that there are certain dipeptides, the occurrence of which is significantly different in un- } \\
\text { stable proteins compared with those in stable ones (12). A protein whose index is }<40 \text { is predicted stable, a value }>40 \text { predicts that } \\
\text { the protein may be unstable. }\end{array}$}} \\
\hline & & & & & & & & & & \\
\hline \multicolumn{11}{|c|}{ 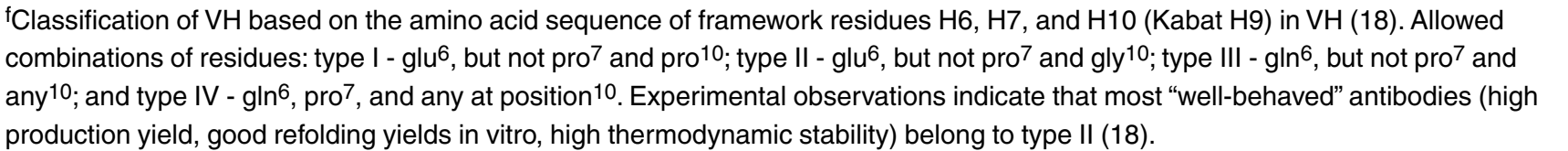 } \\
\hline
\end{tabular}


Table 2. Comparison of the Biophysical Properties of scFv Molecules Based on Different Frameworks

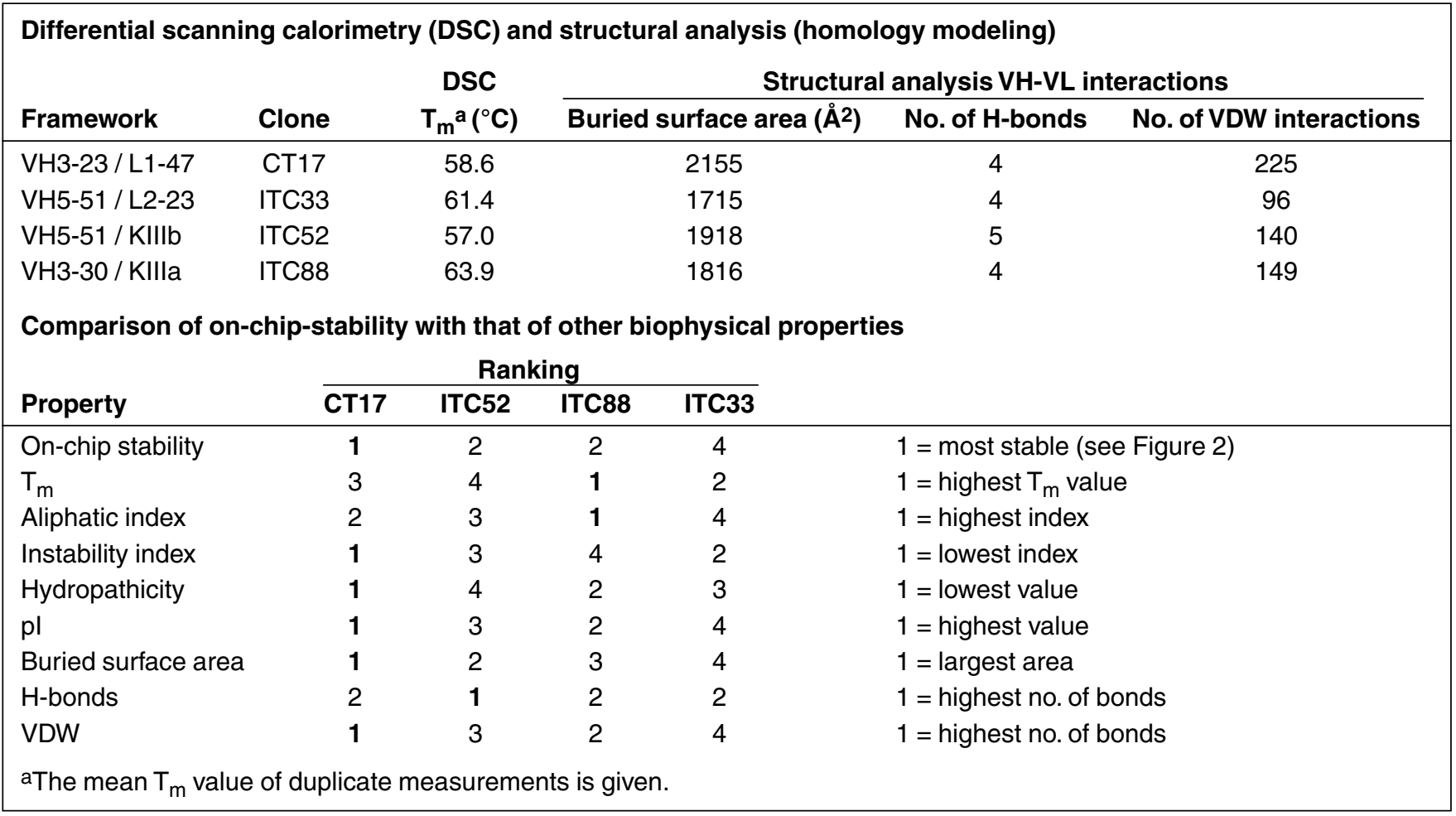

VH3-23/VL1-47 framework, FITC8 and SMUC159. The results showed that FITC8 retained $75 \%$ and SMUC159 70\% of their original antigen-binding activities on day 47 , with predicted half lives of 125 days and 120 days, respectively. To the best of our knowledge, the observed probe stability for the VH323/VL1-47 framework is the highest so far, using the recombinant antibody design based on a single framework.

Recently, it has been shown that the addition of certain chemical agents such as glycerol to the spotting solution may further enhance probe stability $(17,24)$. It should be noted that we used PBS alone as spotting buffer (Figure 2); thus, we may improve probe stability even further by exploring the use of various protective agents. Our protein chips were successfully stored under dry conditions, in accordance with observations that high moisture levels may negatively affect the chemical stability of proteins compared to the situation in the solid state $(4,21)$. Further experiments are, however, required to elucidate whether other storage procedures (e.g., freezing) would prolong protein chip shelf-life even longer.

To gain insights into the different molecular stability of the frameworks, the thermal stability of the $s c F v$ antibodies was determined by differential scanning calorimetry (DSC) and expressed as melting temperature $\left(\mathrm{T}_{\mathrm{m}}\right)$ values (Table $\left.2 \mathrm{~A}\right)$. The results showed that the $\mathrm{T}_{\mathrm{m}}$ values of the frameworks decreased in the order of ITC88 > ITC33 > CT17 > ITC52 (Table 2). Notably, the $T_{m}$ values correlate poorly with the predicted stability of the frameworks based on primary structure analysis (Tables 1 and 2) and with the observed on-chip stability (Figure 2, Table 2). This notion was further supported by DSC analysis of an additional six scFv antibodies based on the VH3-23/VL1-47 framework (clones FITC8, sMUC159, IL-1 $\alpha-I 22$, TNF $\beta-10$,
VEGF-3, and MCP4-6), the $T_{m}$ values of which varied between $61.0^{\circ}$ to $78.0^{\circ} \mathrm{C}$ (data not shown). Taken together, the results showed that the molecular requirements for thermal stability in solution clearly differed from those providing functional stability on a solid support in a dry state. In this context, it should be noted that in the process of stability engineering proteins, improved thermal stability is commonly used as a major selection criteria (35). In the case of engineering probes for protein chips, our results suggest that other screening procedures should be used.

Finally, structural analysis by homology modeling was performed in an attempt to identify structural parameters crucial for the on-chip probe stability (Table 2, Figure 3). The analyses focused on the VH-VL interface, which is considered to be a major weak point of $s c F v$ with respect to stability $(34,35)$. The buried surface area (the contact area of VH-VL interaction) was found to vary from 2155 (CT17) to $1715 \AA^{2}$ (ITC33). While the number of interdomain hydrogen bonds is almost constant, the number of van der Waal (vdw) interactions ranges between 225 (CT17) to 96 (ITC33). No interdomain ionic bonds were detected. In Figure 3, the residues responsible for the vdw interactions are mapped in red onto the structure of the most (CT17) and least stable framework (ITC33) (Figure 3). The main differences, marked by arrows (Figure 3), are located in both ends of the interface. Although weak, vdw interactions contribute significantly to (protein) interdomain stability by providing higher packing efficiency (16). This is further illustrated in Figure 3C, where the structures of CT17 and ITC33 are superimposed. The relative orientation of the domains also differ somewhat, providing a more exquisite fit in the case of CT17. Taken together, the results of this analysis show that two molecular parameters, the buried surface area and number of interdomain vdw interactions, 
correlate well with the on-chip stability (Table 2).

In conclusion, we have shown that the recombinant $\mathrm{scFv}$ antibody design based on a single framework displays superior biophysical parameters, particularly molecular (functional) stability, making them good probe candidates for high-density antibody arrays. The actual choice of framework was, however, absolutely crucial because the adsorption properties and the intrinsic stability of the frameworks differs significantly. The VH3-23/VL147 framework was identified as the superior scaffold for recombinant antibody probes used in microarrays. We believe that recombinant scFv antibodies selected from large molecular libraries will constitute one of the major sources for probes intended for global proteome analysis in the near future. If so, the performance of selected scFvs would improve significantly if the library was based on the single framework approach $(3,31)$. In certain applications, this would even be necessary.

\section{ACKNOWLEDGMENT}

This investigation was supported by grants from the National Council for Engineering Sciences and from SWEGENE.

\section{REFERENCES}

1.Alizadeh, A.A., M.B. Eisen, R.E. Davis, C. Ma, I.S. Lossos, A. Rosenwald, J.C. Boldrick, H. Sabet, et al. 2000. Distinct types of diffuse large B-cell lymphoma identified by gene expression profiling. Nature 403:503-511.

2.Borrebaeck, C.A.K. 2000. Antibodies in diagnostics-from immunoassay to protein chips. Immunol. Today 21: 379-382.

3.Borrebaeck, C.A.K., S. Ekström, A.C. Malmborg Hager, J. Nilsson, T. Laurell, and G. Marko-Varga. 2001. Protein chips based on recombinant antibody fragments: a highly sensitive approach as detected by mass spectrometry. BioTechniques 30:1126-1132.

4.Breen, E.D., J.G. Curley, D.E. Overcashier, C.C. Hsu, and S.J. Shire. 2001 Effect of moisture on the stability of a lyophilized humanized monoclonal antibody formulation. Pharm. Res. 18:1345-1353.

5.Butler, J.E. 2000. Solid supports for enzyme-linked immunosorbent assay and other solid-phase immunoassays. Methods 22:4-23.

6.Chothia, C., I. Gelfand, and A. Kister. 1998. Structural determinants in the sequences of immunoglobulin variable domain. J. Mol. Biol. 278:457-479.

7.Connolly, M.L. 1983. Solvent-accessible surfaces of proteins and nucleic acids. Science 221:709-713.

8.Ek, S., C.-M. Högerkorp, M. Dictor, M. Ehinger, and C.A.K. Borrebaeck 2002. Mantle cell lymphomas express a distinct genetic signature affecting lymphocyte trafficking and growth regulation as compared to normal human B cells. Cancer Res. 62:4398-4405.

9.Glynne, R., G. Ghandour, J. Rayner, D.H. Mack, and C.C. Goodnow. 2000 B-lymphocyte quiescence, tolerance and activation as viewed by global gene expression profiling on microarrays. Immunol. Rev. 176:216-246.

10.Golub, T.R., D.K. Slonim, P. Tamayo, C. Huard, M. Gaasenbeek, J.P. Mesirov, H. Coller, M.L. Loh, et al. 1999. Molecular classification of cancer: class discovery and class prediction by gene expression monitoring. Science 286:531-537.

11.Guex, N. and M.C. Peitsch. 1997. SWISS-MODEL and the Swiss-Pdb Viewer: an environment for comparative protein modeling. Electrophoresis 18:2741-2723.

12.Guruprasad, K., B.V. Reddy, and M.W. Pandit. 1990. Correlation between stability of a protein and its dipeptide composition: a novel approach for predicting in vivo stability of a protein from its primary sequence. Protein Eng. 4:155-161

13.Haab, B.B. 2001. Advances in protein microarray technology for protein expression and interaction profiling. Curr. Opin. Drug Discov. Devel. 4:116-123.

14.Haab, B.B., M.J. Dunham, and P.O. Brown, 2001. Protein microarrays for highly parallel detection and quantitation of specific proteins and antibodies in complex solutions. Genome Biol. 2:RESEARCH0004.1-13.
15.Ikai, A. 1980. Thermostability and aliphatic index of globular proteins. J. Biochem. 88:1895-1898.

16.Jaenicke, R. 2000. Stability and stabilization of globular proteins in solution. J. Biotechnol. 79:193-203.

17.Joos, T.O., M. Schrenk, P. Hopfl, K. Kroger, U. Chowdhury, D. Stoll, D. Schorner, M. Durr, et al. 2000. A microarray enzyme-linked immunosorbent assay for autoimmune diagnostics. Electrophoresis 21:2641-2650.

18.Jung, S., S. Spinelli, B. Schimmele, A. Honegger, L. Pugliese, C. Cambillau, and A. Plückthun. 2001. The importance of framework residues H6, H7 and H10 in antibody heavy chains: experimental evidence for a new structural subclassification of antibody VH domains. J. Mol. Biol. 309:701-716.

19.Klein, U., Y. Tu, G.A. Stolovitzky, I.M. Mattiol, G. Cattoretti, H. Husson, A. Freedman, G. Inghirami, et al. 2001. Gene expression profiling of B cell chronic lymphocytic leukemia reveals a homogeneous phenotype related to memory B cells. Exp. Med. 194:1625-1638.

20.Kyte, J. and R.F. Doolittle. 1982. A simple method for displaying the hydropathic character of a protein. J. Mol. Biol. 157:105-132.

21.Lai, M.C. and E.M. Topp. 1999. Solid-state chemical stability of proteins and peptides. J. Pharm. Sci. 88:489-500.

22.Lantto, J., Y. Lindroth, and M. Ohlin. 2002. Non-germ-line encoded residues are critical for effective antibody recognition of a poorly immunogenic neutralization epitope on glycoprotein B of human cytomegalovirus. Eur. J. Immunol. 32:1659-1669.

23.Lockhart, D.J. and E.A. Winzeler. Genomics, gene expression and DNA arrays. 2000. Genomics, gene expression and DNA arrays. Nature 405:827-836.

24.MacBeath, G. and S.L. Schreiber. 2000. Printing proteins as microarrays for high-throughput function determination. Science 289:1760-1763.

25.McCafferty, J., A.D. Griffiths, G. Winter, and D.J. Chiswell. 1990. Phage antibodies: filamentous phage displaying antibody variable domains. Nature 348:552-554.

26.Mitchell, P. 2002. A perspective on protein microarrays. Nat. Biotechnol. 20:225-229.

27.Nicholls, A., K. Sharp, and B. Honig. 1991. Protein folding and association: insights from the interfacial and thermodynamic properties of hydrocarbons. Proteins 11:281-296.

28.Ohlin, M., H. Owman, J.D. Rioux, M.M. Newkirk, and C.A.K. Borrebaeck. 1994. Restricted variable region gene usage and possible rheumatoid factor relationship among human monoclonal antibodies specific for the $\mathrm{AD}-1$ epitope on cytomegalovirus glycoprotein B. Mol. Immunol. 31:983-991.

29.Spaete, R.R. 1991. A recombinant subunit vaccine approach to HCMV vaccine development. Transplant Proc. 23:90-96.

30.Staudt, L.M. 2001. Gene expression physiology and pathophysiology of the immune system. Trends Immunol. 22:35-40.

31.Söderlind, E., L. Strandberg, P. Jirholt, N. Kobayashi, V. Alexeiva, A.M. Åberg., A. Nilsson, B. Jansson, et al. 2000. Recombining germline-derived CDR sequences for creating diverse single-framework antibody libraries. Nat. Biotechnol. 18:852-856.

32.Webster, D.M., A.H. Henry, and A.R. Rees. 1994. Antibody-antigen interactions. Curr. Opin. Struct. Biol. 4:123-129.

33.Winter, G. and C. Milstein. 1991. Man-made antibodies. Nature 349:293299.

34.Wörn, A. and A. Plückthun. 1999. Different equilibrium stability behaviour of scFv fragments: identification, classification, and improvements by protein engineering. Biochemistry 38:8739-8750.

35.Wörn, A. and A. Plückthun. 2001. Stability engineering of antibody singlechain Fv fragments. J. Mol. Biol. 305:989-1010.

\section{Address correspondence to:}

Dr. Carl A.K. Borrebaeck

Department of Immunotechnology

Lund University

P.O.Box 7031

S-220 07 Lund, Sweden

e-mail:Carl.Borrebaeck@immun.lth.se 